\title{
EPIDEMIOLOGICAL AND CLINICAL CHARACTERISTICS OF 2019 NOVEL CORONA VIRUS: A REVIEW
}

\author{
T SAI LAKSHMI SRIKALA ${ }^{1 *}$, T PRAVEEN KUMAR ${ }^{2}$
}

${ }^{1}$ Department of Pharmacy Practice, Anurag Pharmacy College, Kodad, Suryapet, Telangana, India. ${ }^{2}$ Department of Pharmaceutics, Anurag Pharmacy College, Kodad, Suryapet, Telangana, India. Email: srichowdary.chinni33@gmail.com

Received: , Revised and Accepted:

\section{ABSTRACT}

Middle East Respiratory Syndrome (MERS), having a high demise rate, which radiate from the Middle East. Novel Coronavirus is the causative living being for MERS and (2019-nCoV) was recognized infection by Chinese experts on January 7. On December 31, 2019, the WHO China Country Office was educated regarding instances of pneumonia of obscure etiologic (obscure reason) identified in Wuhan City, Hubei Province of China. Besides, MERS-CoV first found in dromedary camels and in bats, people. Proof recommends that animals are acting as host for MERS-CoV and transmitting the malady to people. Till the finish of November 2019, an aggregate of 2494 lab instances of MERS, were recorded which incorporates 858 deaths around the world. At present, no drug or immunization is accessible for MERS. As we knew that prevention is better than cure, taking precautions before attacking of the disease is the best way of preventing disease. In this study, we discussed about the pathological aspects, treatment strategies, and precautions of the disease.

Keywords: Coronavirus, ELISA, Monoclonal antibodies, Dromedary camels, WHO, Wuhan City.

(C) 2021 The Authors. Published by Innovare Academic Sciences Pvt Ltd. This is an open access article under the CC BY license (http://creativecommons.org/ licenses/by/4.0/) DOI: http://dx.doi.org/10.22159/ijs.2021v9i6.40448. Journal homepage: https://innovareacademics.in/journals/index.php/ijs

\section{INTRODUCTION}

Coronaviruses (CoV) are an enormous group of infections which prompts conditions from the regular virus to exceptionally serious ailments such as Middle East Respiratory Syndrome (MERS-CoV) and Severe Acute Respiratory Syndrome (SARS-CoV). The new strain of infection which currently recognized in people is novel $\mathrm{CoV}$ (nCoV). CoVs is zoonotic in nature, which implies that they are proficient to transmit from creatures to people. Proof uncovers that civet cats transmit SARSCoV TO people and dromedary camels transmit MERS-CoV to people. There are many known CoVs that are available in creatures that have not yet contaminated people. Scarcely any instances of pneumonia of unknown etiology (obscure reason) were distinguished in Wuhan City, Hubei Province of China educated by WHO china nation office on December 31, 2019.

On January 7, Chinese specialists recognized nCoV (2019-nCoV) as the causative life form on January 10, the WHO distributed a scope of direction for all nations on the best way to be set up for the infection, includes monitoring sick people, test samples, treat patients, infection control, maintain the right supplies, and creating awareness in the public about this new virus. Nations are urged to keep fortifying their readiness for new malady flare-ups in accordance with the International Health Regulations (2005). Standard suggestions for forestalling contamination spread incorporates washing hands routinely, while hacking and wheezing spread mouth and nose, eat meat, and eggs which are altogether cooked. Evade close contact with individuals indicating manifestations of respiratory ailment, for example, hacking and sneezing [1]

\section{EPIDEMIOLOGY}

- 2229 research center affirmed instances of MERS-CoV disease were accounted for the WHO somewhere in the range of 2012 and 30 June 2018, Kingdom of Saudi Arabia detailed 83\% among those. Complete cases have been accounted for from 27 nations in the Middle East, North Africa, Europe, the United States of America, and Asia

- Since the keep going worldwide update distributed on July 21 2017, four nations were accounted for 189 research center affirmed instances of MERS-CoV to the WHO (182 from Saudi Arabia, three from Oman, three from the United Arab Emirates, and one from Malaysia), of which 60 (31.7\%) have kicked the bucket. Among them, $75.5 \%$ were male and the middle age was 54 years [3]

- 2494 research center affirmed instances of MERS, were accounted for before the finish of November 2019 which incorporates 858 passing's (case-casualty rate: $34.4 \%$ ); most of these cases were accounted for from Saudi Arabia $(2102$ cases, incorporating 780 related deaths with a case-casualty pace of $37.1 \%$ )

- Individuals of age group 50-59 years have the most noteworthy hazard for getting disease of essential cases. The age bunch 30-39 years is most in danger for auxiliary cases. The quantity of passing's is higher in the age bunch 50-59 years for essential cases and 70-79 years for auxiliary cases [2]. Epidemiological status of corona virus is shown on Fig. 1.

\section{TRANSMISSION AND PATHOGENESIS OF MERS-COV}

MERS-CoV disease was at first notion to spread by zoonotic occasions by means of bats as phylogenetic examinations uncovered that it is hereditarily associated with Tylonycteris bat CoV HKU4 (BatCoVHKU4) and Pipistrelle's bat CoV HKU5 (BatCoV-HKU5) [4].

Nonetheless, proof demonstrates that MERS-CoV started from dromedary camels. A serological report proposes that practically $90 \%$ of all camels in Africa and the Middle East were seropositive for MERS$\mathrm{CoV}$, where different animals, for example, sheep, goats, and dairy animals were discovered negative $[5,6]$.

Furthermore, antibodies against MERS-CoV were found in tests acquired from camels in Saudi Arabia in 1993, which fortifies the theory that dromedary camels are doubtlessly the primary repositories of MERS-CoV. Conversely, no xenoreactivities detailed in the blood tests got from blood benefactors and abattoir laborers in Saudi Arabia during 2012. MERS-CoV identified in camels in Egypt that were privately raised or imported from nations where no MERS cases revealed [7]. The method of transmission is as yet obscure, yet it suspected to through saliva or during direct contact with contaminated camels or through utilization of milk or uncooked 


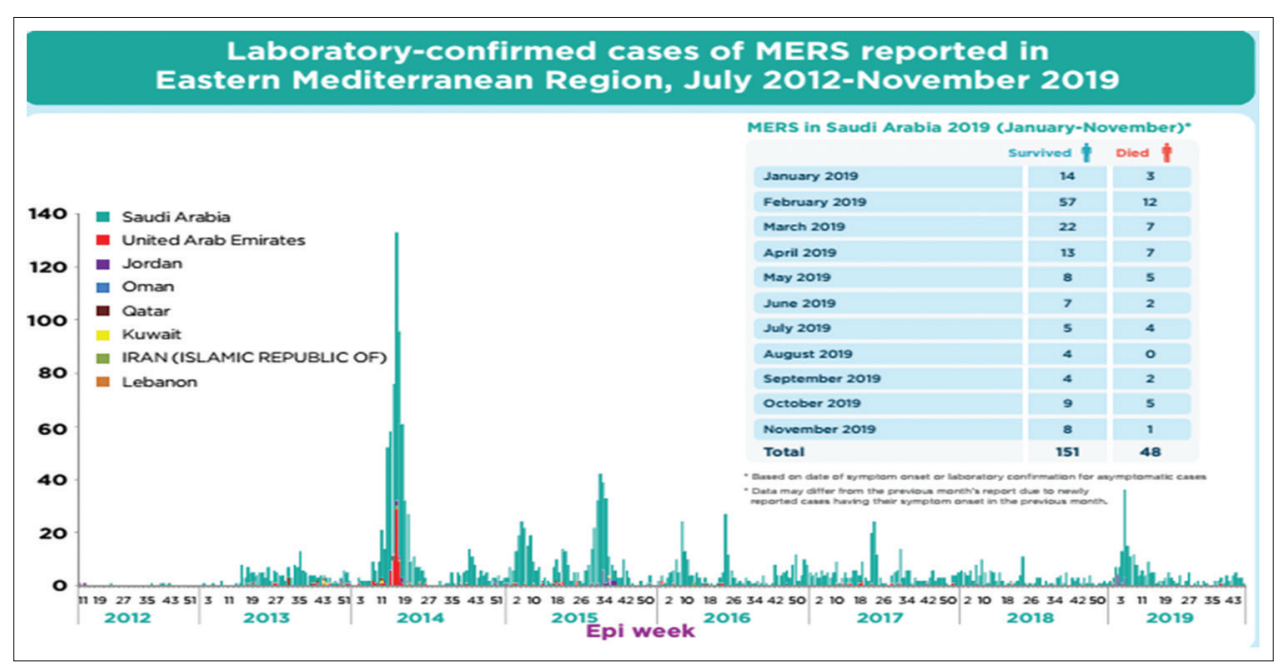

Fig. 1: Epidemiological status of Corona virus in population from 2012 to 2019 [2]

meat. Nonetheless, we cannot preclude with exist of another middle of the road have for MERS-CoV transmission to people [7].

Auxiliary contamination may happen through droplets or contact, and the infection could spread either by means of air or fomites [8]. A couple of ongoing investigations on tainted patients demonstrated that the most widely recognized MERS-CoV contamination causes intense pneumonia and renal disappointment and that pretty much every patient created respiratory issues. Furthermore, at any rate $33 \%$ of the contemplated patients were additionally answered to have stomach issue. Different impacts incorporate aggravation of the pericardium, immoderate coagulopathy, increment in leukocytes and neutrophils, and low degrees of lymphocytes, platelets and red blood cells. Besides, hyponatremia and low blood levels of albumin recognized during the contextual analysis $[9,10]$.

\section{ENTRY AND REPLICATION OF MERS-COV IN THE HOST CELL}

A diagram of the section and replication procedure of MERS-CoV in the tainted host cell is appeared in Fig. 2 and examined underneath. The S glycoprotein situates on the outside of the Tropism and receptor collaboration, whereas membrane-fusing mediators was recognized inside the S2 area [11,12]. MERS-CoV ties to dipeptidyl peptides 4 (DPP4) through a receptor-binding domain (RBD) in the S1 subunit.

Therefore, protease cleavage of the $S$ protein prompts infection cell combination and the arrival of viral genomic RNA into the host cytoplasm [13,14]. The main interpretation starts in ORF1a and proceeds in ORF1b after a frame shift, along these lines delivering the polyproteins pp1a and pp1ab, individually $[15,16]$.

ORF $1 \mathrm{ab}$, which contains $66 \%$ of the genome, is answerable for encoding non-basic proteins (nsps), since the staying 33\% of the genome encodes basic proteins (E, N, S and M) and five frill proteins [15]. The infection encoded proteases PLpro and 3CLpro sever the pp1a and pp1ab proteins at three and 11 unique locales, individually, bringing about 16 develop nsps [17]. The proteins engaged with replication and interpretation (RNA-subordinate RNA polymerase and helicase encoded by ORF1ab) structure replication-translation buildings [16]. These complexes assemble at the perinuclear districts and partner with double membrane vesicles got from the endoplasmic reticulum (ER) [8] It has affirmed through electron tomography and three-dimensional recreation imaging of the SARS-CoV-contaminated Vero E6 cells that double membrane vesicles are not separate vesicles however are rather part of a reticulo vesicular arrangement of modified ER layers [18].

The genomic RNA has adenylate uridylate (AU)-rich groupings considered interpretation guideline arrangements that are around ten nucleotides in length; these successions isolate the genomic RNA into various body components of different lengths. These interpretation guideline arrangements are either perceived by the replication-translation edifices to create suspended short negative-strand RNA of sub genomic length on the transcript of adornment and basic proteins or persistent fulllength less strand layout RNA of genomic length for replication [16]. The recently integrated genomic RNAs are typified in the $\mathrm{N}$ proteins in the cytoplasm and afterward shipped to the ER-Golgi halfway compartment for additional get together. The proteins S, M, and E were embedded into the layer of the rough ER and are then shipped to the ER-Golgi intermediate compartment where they communicate with the $\mathrm{N}$ proteins and gather into particles. The grown vesicles are then moved to the cell surface for discharge after development in Golgi bodies [8,18].

In the existence pattern of RNA infections, interferons (IFNs) have a pivotal job in the counter popular protection and they were initiated by double stranded RNAs (dsRNAs) created during viral replication [19]. Golgi intermediate compartment including Toll-like receptors (TLRs) and retinoic acid inducible gene I (RIG-I)-like receptors (RIG-I and melanoma differentiation-associated protein 5 [MDA5]) has a basic job in natural resistance [20-22].

Among TLRs, TLR3 explicitly perceives dsRNAs through its ecto space [23] while RIG-I and MDA5 additionally perceive dsRNAs through their helicase domain [20]. In the wake of detecting dsRNAs, activated immune response induces IFNs and cytokines to block viral replication. To evade this cellular immune response, MERS-CoV 4 a protein binds to dsRNAs and obstructs the induction of type 1 IFN [24,25].

MERS-CoV ties to DPP4 on the host cell through its RBD in the S1 subunit of the spike (S) glycoprotein, which prompts infection cell combination and the arrival of genomic RNA into the cytoplasm. At first open perusing outline 1a (ORF1a) and ORF1b was converted into polyproteins, polyprotein $1 \mathrm{a}$ (pp1a) and pp1ab, separately, which is divided by the infection encoded proteases papain-like protease (PLpro) and 3C-like protease (3CLpro) into 16 develop nsps.

The proteins engaged with replication and interpretation is accumulated into replication-translation complexes (RTCs) that partner with double-membrane vesicles got from the ER. The genomic RNA has AU- rich groupings called transcription regulation sequences (TRSs). On the off chance that the TRSs was perceived by RTCs, at that point RNA of sub genomic length for interpretation has been created, in any case a full-length format RNA of genomic length for replication has been orchestrated. The recently created genomic RNAs are typified in the nucleo capsid $(\mathrm{N})$ proteins in the cytoplasm and afterward moved to the ER-Golgi intermediate compartment (ERGIC) for additional gathering. The $\mathrm{S}$, layer (M), and envelope (E) proteins are embedded 


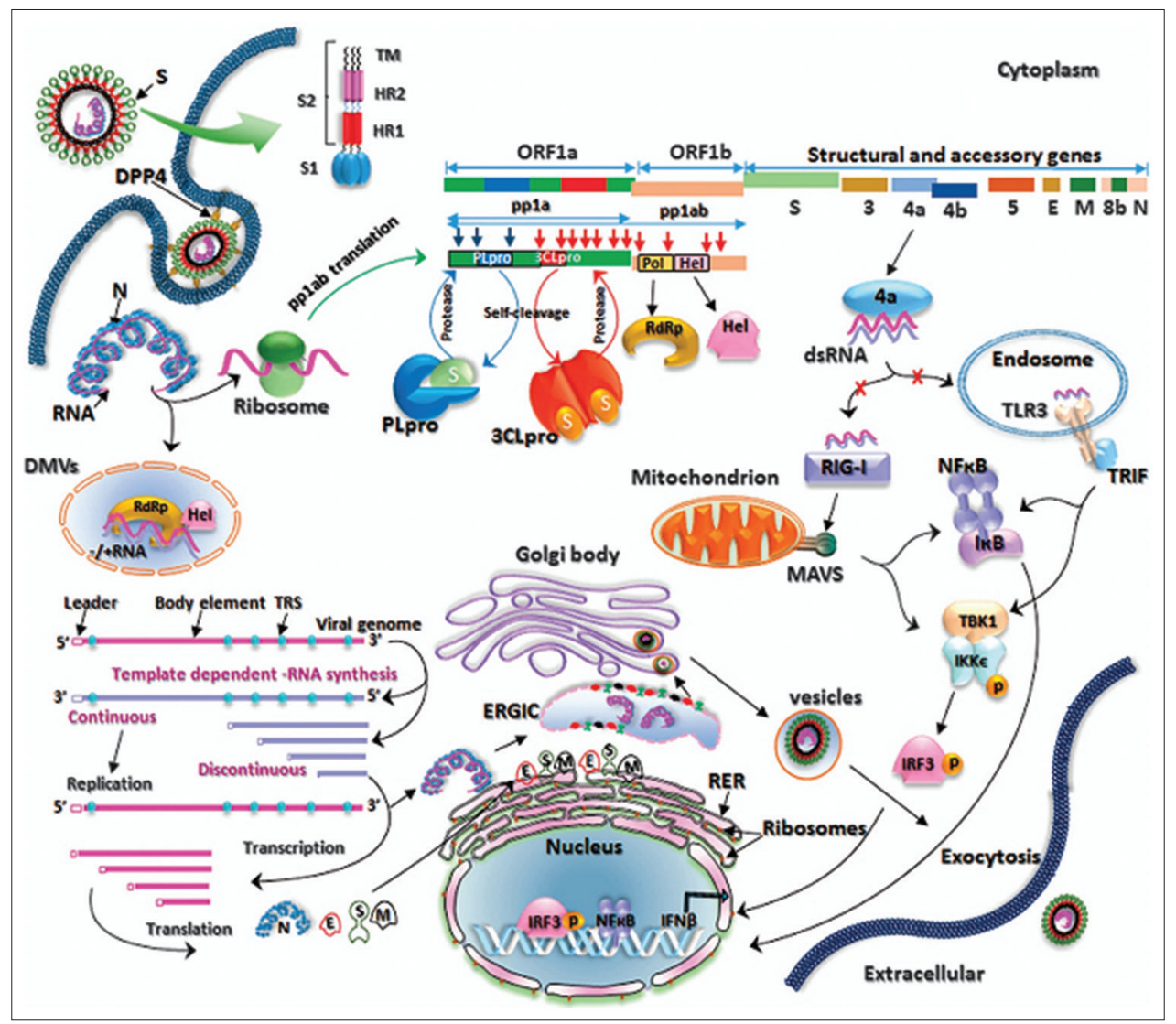

Fig. 2: Schematic of the replication pattern of Middle East respiratory syndrome coronavirus

into the film of the unpleasant ER (RER), from where they were shipped to the ERGIC to collaborate with the RNA-exemplified N proteins and collect into viral particles.

The grown vesicles containing experienced viral particles are then moved to the cell surface for discharge after development in the Golgi bodies. dsRNAs are incompletely created during viral replication. The 4a contends with TLR3 and retinoic corrosive inducible quality I item (RIG-I)- like helicases (RIG-I and MDA5) to tie to dsRNAs and evades the host immune response. Schematic of the replication pattern of Middle East respiratory syndrome corona virus is shown in Fig. 2.

\section{DIAGNOSIS}

On a whole, there are two classifications of research center tests:

- Molecular tests-these identifies the active disease

- Serology tests-these are equipped for identifying past infection by recognizing antibodies to MERS-CoV. This test is predominantly utilized for surveillance or investigational purposes and not for analytic employments.

\section{MOLECULAR TESTS}

In individuals who are believed to be contaminated with MERS$\mathrm{CoV}$, active disease can be analyzed by molecular tests individuals' dependent on their clinical manifestations.

Real-time reverse-transcription polymerase chain response (rRT-PCR) examines are atomic tests that can be utilized to identify viral RNA in clinical samples. CDC's present case definition for research center affirmation of MERS-CoV contamination requires either a positive rRTPCR result for at any rate two explicit genomic targets, or a solitary positive objective with sequencing of a subsequent objective.

- Most state research facilities in the united states are endorsed to test for MERS-CoV by utilizing a rRT-PCR test created by CDC. This test is done under power of an Emergency Use Authorization in light of the fact that there are no FDA-cleared/affirmed tests accessible for this reason in the United States

- The accomplishment of rRT-PCR testing relies on a few factors, including the experience and mastery of research facility work force, lab condition (e.g., evasion of defilement), and the sort and state of examples being tried. For this rRT-PCR examine, CDC suggests gathering various examples, including lower (bronchoalveolar lavage, sputum and tracheal suctions) and upper (e.g., nasopharyngeal and oropharyngeal swabs) respiratory examples, serum, and stool examples

- $\quad$ CDC believes a patient under scrutiny to be negative for dynamic MERS-CoV infection tailing one negative rRT-PCR test on the suggested examples. Since a solitary negative outcome does not totally preclude MERS-CoV disease, in certain conditions extra examples might be tried.

\section{SEROLOGY TESTS}

Serology testing is utilized to identify past infection (antibodies to MERS-CoV) in individuals who may have been presented to the infection. Antibodies are proteins created by the body's invulnerable framework to defend and kill infections, bacteria, and different microorganisms during contamination. The nearness of antibodies to MERS-CoV shows that an individual had been recently contaminated with the infection and built up an insusceptible reaction.

- Evidence to date proposes there might be a more extensive scope of MERS infection than was at first idea. For instance, general well-being examiners have distinguished people who are PCRconstructive, however, have no MERS indications; we do not have a clue whether MERS-CoV can be spread by these individuals. Thus, general wellbeing researchers are attempting to become familiar with how the infection is transmitted. One approach to do this is through deliberate testing of blood tests from individuals who had close contact with individuals known to have MERS 
- $\quad$ CDC has a two-stage approach for serology testing, utilizing two screening tests and one corroborative test to identify antibodies to MERS-CoV

- ELISA, enzyme-linked immunosorbent assay, is a screening test used to recognize the nearness and convergence of explicit antibodies that bind to a viral protein. CDC tests by ELISAS for antibodies against two diverse MERS-CoV proteins, the nucleocapsid (N) and spike (S)

- If a clinical example is resolved to be immune response positive by either ELISA, CDC at that point utilizes the micro neutralization test to affirm the positive outcome

- The micro neutralization test is an exceptionally explicit corroborative test used to gauge killing antibodies, or antibodies that can kill infection. This technique is viewed as a best quality level for recognition of explicit antibodies in serum tests. In any case, contrasted and the ELISA, the micro neutralization test is work serious and tedious, requiring at any rate 5 days before results are accessible

- If a clinical example is sure by either ELISA, or positive by micro neutralization test, the example is resolved to be affirmed positive

- If a clinical example is sure by the two ELISAs, and negative by micro neutralization test, the example is resolved to be vague

- If a clinical sample is certain by just a single ELISA, and negative by micro neutralization test, the example is resolved to be negative

- If a clinical sample is negative by the two ELISAS, the example is resolved negative

- In the end, a last assurance of an affirmed positive serology result requires a positive ELISA test and affirmation by micro neutralization test

- MERS-CoV serology tests are for reconnaissance or investigational purposes and not for analytic purposes - they are apparatuses created in light of the MERS-CoV episode

- Information is constrained about MERS-CoV and how the infection is spread. As general wellbeing researchers become familiar with MERS-CoV, the way to deal with leading these kinds of lab tests may change [26].

\section{TREATMENT}

Despite past efforts to develop CoV countermeasures, there are still no licensed therapies of proven efficacy for MERS-CoV or any other CoV infection. Supportive measures remain the mainstay of MERS-CoV treatment strategies and include respiratory and circulatory support, preservation of renal, hepatic and neurological function, and prevention of secondary infections. There are animal data where combination treatment of ribavirin and IFN- $\alpha 2 \mathrm{~b}$ improved therapeutic outcomes in MERS-CoV-infected non-human primates. However, treatment was initiated very soon after viral challenge $(-8 \mathrm{~h})$, a window that is unlikely to be replicated in a real-world clinical setting [27].

Ribavirin, for example, is a potent nucleoside analogue that has been used with varying measures of success against a range of RNA viruses [28]. However, patients can experience significant toxicities when given the drug alone or in combination with an IFN, including but not limited to hemolytic anemia and metabolic abnormalities [29].

Rapidly scaled treatments based on naturally occurring neutralizing antibodies such as convalescent plasma or hyper immune globulin, on the other hand, have been shown to be relatively safe and potentially effective for reducing mortality from several infections such as SARS$\mathrm{CoV}$ and influenza and may hold promise for MERS-CoV as well [30-32].

One study found that convalescent plasma decreased mortality in SARS$\mathrm{CoV}$ patients only if administered within 14 days of illness [31]

\section{MONOCLONAL ANTIBODIES (mAbs)}

mAbs, on the other hand, can be administered in the setting of an outbreak without the need to discriminate who might be at greatest risk for infection. During early in their natural history and for post-exposure prophylaxis of case these can be used. mAbs also carry the benefits of higher potency, greater specificity, more extensive pre-licensing evaluation, and consequently a more vetted safety profile. mAbs also helps in defining immunogenic epitopes through crystallographic analysis, and provides atomic-level detail for the design of better immunogens. It is proved that they are effective therapies in the areas of cancer management and autoimmune disease management.

Although there is only one pathogen, respiratory syncytial virus, for which a mAb is licensed for use, there are a number of other infectious disease indications - such as Ebola virus disease treatment and human immunodeficiency virus primary and secondary prevention. Almost all of the published mAbs and all of those in development target the $S$ RBD, which contains the most immunogenic epitopes on the virus. Many bind to the RBD, expressed both on a recombinant $S$ and on the surface of live virus, with picomolar affinity and neutralize MERS-CoV at a half maximal inhibitory concentration (IC50)of $10 \mathrm{ng} / \mu \mathrm{L}$ or less [33].

Although intensive, supportive care still serves as the primary treatment option for MERS-CoV there are few new antivirals for MERS-CoV, one in particular, GS-5734 developed by Gilead Sciences, is an adenine analogue that is incorporated into viral RNA to disrupt replication [34].

Similarly, BCX4430 is a nucleoside analogue that is being developed by Biocryst Pharmaceuticals for potential treatment of filoviruses, coronaviruses and other RNA viruses [35]

Mycophenolic acid, an inhibitor of both T an B lymphocytes, has also been found to have strong activity against MERS-CoV, as it does against other RNA viruses such as West Nile, hepatitis $C$ and dengue [36].

Lopinavir is the one among the drugs to show in vitro activity against MERS-CoV, as it is tested in an animal model. MERS-CoV-challenged marmosets that were treated with this protease inhibitor had better clinical, pathological, virological, and radiological outcomes than controls or those treated with mycophenolate mofeti [37].

Adding to this, two peptides, HR1P and HR2P are being produced as potential fusion inhibitors. By acting on the six-helix bundle core of the MERS-CoV S protein to prevent protein-mediated cell-to-cell fusion, this class of compounds may hold potential beyond MERS-CoV towards a long-term objective of a pan-coronavirus antiviral [38].

\section{PRECAUTIONS}

\section{General precautions}

- Avoiding contact with camels

- Avoid drinking crude camel milk or camel pee

- Avoid eating camel meat that has not been completely cooked

- Hands ought to be washed with cleanser and water as well as liquor gel after each contact with a corporeal

- Workers should wear facial assurance where attainable; and defensive apparel, which ought to be expelled after work and washed every day

- Workers ought to abstain from presenting relatives to dirtied work garments, shoes, or different things that may have come into contact with camel emissions and discharges. These garments and different things ought to stay at the working environment for day by day washing and laborers ought to approach and utilize shower offices at their work environments before leaving the premises

- Health authorities in nations outside of the influenced locales ought to keep up ant all level of cautiousness, particularly those in nations with huge quantities of voyagers or transient laborers coming back from the Middle East [2].

\section{TRANSMISSION PRECAUTIONS}

MERS-CoV is accepted to spread between people basically through contact and respiratory beads. In any case, transmission through little molecule bead cores (aerosols) may happen. Natural defilement amid episodes in healthcare facilities can be broad and might contributes to 
opening up flare-ups, on the off chance that satisfactory disinfection methods are not taken after.

- For patients with suspected or affirmed MERS-CoV contamination who are definitely not basically sick, Standard, Contact, and Droplet safety measures are prescribed

- For patients who are basically sick, Standard, Contact, and Airborne safety measures are prescribed because of the high probability of requiring airborne creating methodology.

\section{Patient Precautions}

Patients with suspected or affirmed MERS-CoV contamination who are not basically sick ought to be set in single patient rooms in a territory that is plainly isolated from other patient-care zones. A versatile HEPA channel could be utilized and set concurring to the maker suggestions.

- Critically sick patients with suspected or affirmed MERS-CoV disease ought to be put in airborne infection isolation rooms (negative pressure rooms), if accessible. At the point when negative weight rooms are not accessible, the patients ought to be set in enough ventilated private rooms with a convenient HEPA channel and is put by the maker suggestions

- Patients should wear a careful veil during development to contain emissions

- Use courses of transport that limit exposures of staff, different patients, and guests

- Notify the accepting zone of the patient's finding and essential safeguards as quickly as time permits before the patient's appearance

- Ensure that human services laborers (HCWs) who are moving patients wear suitable PPE and perform hand cleanliness a while later [39].

\section{CONCLUSION}

MERS is caused by nCoV, the infection of 2019-nCoV was of clustering onset, is more likely to infect older men with comorbidities, and can result in severe and even fatal respiratory diseases such as ARDS. Creating awareness of the disease to the public is the responsibility of the Health care professionals. The virus uses its spike proteins as adhesive factors that are pro-inflammatory for host entry through a specific receptor called DPP4 which is regarded as a key factor in the signal transmission and activate the acquired and innate immunity in infected patients.

Further research is needed to achieve a better knowing of the innate immune response to MERS-CoV. To provoke a particular immune response without disease progress, an effective vaccine should be formulated. As it does not have any treatment or vaccination criteria, infected persons and the healthy persons should follow precautions to avoid further disease complications of the disease. Health-care professionals or physicians should provide awareness regarding the disease to the public so that the frequency of spread of disease can be reduced to some extent. Thus, there is immediate need of developing novel MERS-CoV therapies and vaccines to reduce the contamination of the virus from infected patients, there by mitigating the risk of any potential pandemics.

\section{REFERENCES}

1. Available from: https://www.who.int/health-topics/coronavirus

2. Available from: https://www.who.int/emergencies/mers-cov/en

3. World Health Organization. WHO MERS-CoV Global Summary and Assessment of Risk, Geneva, Switzerland: World Health Organization; 2018.

4. Woo PC, Lau SK, Li KS, Tsang AK, Yuen KY. Genetic relatedness of the novel human group $\mathrm{C}$ betacoronavirus to Tylonycteris bat coronavirus HKU4 and Pipistrellus bat coronavirus HKU5. Emerg Microb Infect 2012;1:1-5

5. Kayali G, Peiris M. A more detailed picture of the epidemiology of Middle East respiratory syndrome coronavirus. Lancet Infect Dis 2015;15:495-7.

6. Milne-Price S, Miazgowicz KL, Munster VJ. The emergence of the Middle East respiratory syndrome coronavirus. Pathog Dis
2014;71:121-36.

7. Watson JT, Hall AJ, Erdman DD, Swerdlow DL, Gerber SI. Unraveling the mysteries of Middle East respiratory syndrome coronavirus. Emerg Infect Dis 2014;20:1054.

8. Zumla A, Hui DS, Perlman S. Middle East respiratory syndrome. Lancet 2015;386:995-1007.

9. Chan JF, Lau SK, Woo PC. The emerging novel Middle East respiratory syndrome coronavirus: The "knowns" and "unknowns". J Formosan Med Assoc 2013;112:372-81.

10. WHO MERS-CoV Research Group. State of knowledge and data gaps of Middle East respiratory syndrome coronavirus (MERS-CoV) in humans. PLoS Curr 2013;5:30127ddb8.

11. Weiss SR, Navas-Martin S. Coronavirus pathogenesis and the emerging pathogen severe acute respiratory syndrome coronavirus. Microbiol Mol Biol Rev 2005;69:635-64.

12. Du L, He Y, Zhou Y, Liu S, Zheng BJ, Jiang S. The spike protein of SARS-CoV a target for vaccine and therapeutic development. Nat Rev Microbiol 2009;7:226-36.

13. Wang N, Shi X, Jiang L, Zhang S, Wang D, Tong P, et al. Structure of MERS-CoV spike receptor-binding domain complexed with human receptor DPP4. Cell Res 2013;23:986.

14. Matsuyama S, Ujike M, Morikawa S, Tashiro M, Taguchi F. Proteasemediated enhancement of severe acute respiratory syndrome coronavirus infection. Proc Natl Acad Sci 2005;102:12543-7.

15. Perlman S, Netland J. Coronaviruses post-SARS: Update on replication and pathogenesis. Nat Rev Microbiol 2009;7:439-50.

16. Sawicki SG, Sawicki DL, Siddell SG. A contemporary view of coronavirus transcription. J Virol 2007;81:20-9.

17. Lee H, Lei H, Santarsiero BD, Gatuz JL, Cao S, Rice AJ, et al. Inhibitor recognition specificity of MERS-CoV papain-like protease may differ from that of SARS-CoV. ACS Chem Biol 2015;10:1456-65.

18. De Haan CA, Rottier PJ. Molecular interactions in the assembly of coronaviruses. Adv Virus Res 2005;64:165-230.

19. Balachandran S, Roberts PC, Brown LE, Truong H, Pattnaik AK, Archer DR, et al. Essential role for the dsRNA-dependent protein kinase PKR in innate immunity to viral infection. Immunity 2000;13:129-41.

20. Kato H, Takahasi K, Fujita T. RIG-I-like receptors: Cytoplasmic sensors for non-self RNA. Immunol Rev 2011;243:91-8.

21. Anwar MA, Basith S, Choi S. Negative regulatory approaches to the attenuation of Toll-like receptor signaling. Exp Mol Med 2013;45:11.

22. Krishnan J, Selvarajoo K, Tsuchiya M, Lee G, Choi S. Toll-like receptor signal transduction. Exp Mol Med 2007;39:421-38.

23. Alexopoulou L, Holt AC, Medzhitov R, Flavell RA. Recognition of double-stranded RNA and activation of NF-אB by Toll-like receptor 3 . Nature 2001;413:732-8.

24. Niemeyer D, Zillinger T, Muth D, Zielecki F, Horvath G, Suliman T, et al. Middle East respiratory syndrome coronavirus accessory protein 4a is a Type I interferon antagonist. J Virol 2013;87:12489-95.

25. Siu KL, Yeung ML, Kok KH, Yuen KS, Kew C, Lui PY, et al. Middle east respiratory syndrome coronavirus $4 \mathrm{a}$ protein is a double-stranded RNA-binding protein that suppresses PACT-induced activation of RIG-I and MDA5 in the innate antiviral response. J Virol 2014;88:4866-76.

26. Roush S, Fast H, Miner CE, Vins H, Baldy L, McNall R, et al. National Center for Immunization and Respiratory Diseases (NCIRD) Support for Modernization of the Nationally Notifiable Diseases Surveillance System (NNDSS) to Strengthen Public Health Surveillance Infrastructure in the US. In: 2019 CSTE Annual Conference CSTE; 2019.

27. Falzarano D, De Wit E, Rasmussen AL, Feldmann F, Okumura A, Scott DP, et al. Treatment with interferon- $\alpha 2 \mathrm{~b}$ and ribavirin improves outcome in MERS-CoV-infected rhesus macaques. Nat Med 2013;19:1313-7.

28. Gross AE, Bryson ML. Oral ribavirin for the treatment of noninfluenza respiratory viral infections: A systematic review. Ann Pharmacother 2015;49:1125-35

29. Jonasch E, Haluska FG. Interferon in oncological practice: Review of interferon biology, clinical applications, and toxicities. Oncologist 2001;6:34.

30. Arabi Y, Balkhy H, Hajeer AH, Bouchama A, Hayden FG, Al-Omari A, et al. Feasibility, safety, clinical, and laboratory effects of convalescent plasma therapy for patients with Middle East respiratory syndrome coronavirus infection: A study protocol. Springerplus 2015;4:709.

31. Mair-Jenkins J, Saavedra-Campos M, Baillie JK, Cleary P, Khaw FM, Lim WS, et al. The effectiveness of convalescent plasma and hyperimmune immunoglobulin for the treatment of severe acute respiratory infections of viral etiology: A systematic review and exploratory meta-analysis. J Infect Dis 2015;211:80-90.

32. Winkler AM, Koepsell SA. The use of convalescent plasma to treat 
emerging infectious diseases: focus on Ebola virus disease. Curr Opin Hematol 2015;22:521-6.

33. Modjarrad K. Treatment strategies for Middle East respiratory syndrome coronavirus. J Virus Erad 2016;2:1-4

34. Warren T, Jordan R, Lo M, Soloveva V, Ray A, Bannister R, et al. Nucleotide prodrug GS-5734 is a broad-spectrum filovirus inhibitor that provides complete therapeutic protection against the development of Ebola virus disease (EVD) in infected non-human primates. Open Forum Infect Dis 2015;2:LB-2.

35. Warren TK, Wells J, Panchal RG, Stuthman KS, Garza NL, Van Tongeren SA, et al. Protection against filovirus diseases by a novel broad-spectrum nucleoside analogue BCX4430. Nature 2014;508:402-5.

36. Dyall J, Coleman CM, Hart BJ, Venkataraman T, Holbrook MR, Kindrachuk J, et al. Repurposing of clinically developed drugs for treatment of Middle East respiratory syndrome coronavirus infection Antimicrob Agents Chemother 2014;58:4885-93.

37. Chan JF, Yao Y, Yeung ML, Deng W, Bao L, Jia L, et al. Treatment with lopinavir/ritonavir or interferon- $\beta 1 \mathrm{~b}$ improves outcome of MERS-CoV infection in a nonhuman primate model of common marmoset. J Infect Dis 2015;212:1904-13.

38. Gao J, Lu G, Qi J, Li Y, Wu Y, Deng Y, et al. Structure of the fusion core and inhibition of fusion by a heptad repeat peptide derived from the $\mathrm{S}$ protein of Middle East respiratory syndrome corona virus. J Virol 2013;87:13134-40.

39. Command and Control Centre. Middle East Respiratory Syndrome Corona Virus; Guidelines for Healthcare Professionals. Vol. 5. Ministry of Health; 2018. p. 7-9 